\title{
Joint Energy and Route Optimization in MIMO Wireless Sensor Networks
}

\author{
Rong $\mathrm{Yu}^{1}$, Yan Zhang ${ }^{2}$, Lingyang Song ${ }^{3}$, and Wenqing Yao ${ }^{1}$ \\ ${ }^{1}$ South China University of Technology, China \\ ${ }^{2}$ Simula Research Laboratory, Norway; Department of Informatics, University of Oslo, Norway \\ ${ }^{3}$ Peking University, China
}

\begin{abstract}
In this paper, we study the reliable packet forwarding in Wireless Sensor Networks (WSNs) with the multipleinput multiple-output (MIMO) and orthogonal space time block codes (OSTBC) techniques. The objective is to propose a crosslayer optimized forwarding scheme to maximize the Successful Transmission Rate (STR) while satisfying the given end-toend energy consumption constraint. The channel coding, energy allocation, and route planning are jointly considered to improve the STR. After formulating the problem as a local stochastic optimization, we propose a low-complexity distributed scheme to reduce the implementation complexity. We show by simulations that the proposed scheme can achieve much higher STR in comparison with existed energy-efficient routing protocols, in which no joint design is considered.
\end{abstract}

\section{INTRODUCTION}

In many applications of wireless sensor networks (WSNs), reliable end-to-end transmissions are crucial. In these applications, the failures of end-to-end transmission of critical information could directly lead to tremendous loss. Since sensors are usually assigned to fulfill the data collection from the source to the data concentration center following a multihop route, the Symbol Error Rate (SER) of the end-to-end transmissions is an important parameter that reflects the reliability. One effective way to improve the reliability is to minimize the source-to-destination (S-D) SER [or equivalently, to maximize the Successful Transmission Rate (STR)]. Besides the reliability issue, energy efficiency is another key concern in WSNs [1]. Since sensors are typically battery-powered, their operation time is stringently limited by the battery capacity. To prolong the lifetime of WSNs, energy resource on sensors should be carefully managed. In this paper, we simultaneously take into account the issues of transmission reliability and energy conservation. Our objective is to maximize the endto-end STR while satisfying the given energy constraint.

It is well known that the multiple-input multiple-output (MIMO) techniques improve system performance and capacity. Thus, it would be desirable to deploy MIMO and apply orthogonal space-time block codes (OSTBC) [2] in WSNs for spatial and temporal diversities in a fading environment. Furthermore, [3] has shown that in many cases the power consumption in the electronic circuit of a MIMO system is more efficient than that in a single-input single-output system (SISO). Hence, from energy efficiency point of view, it is beneficial to implement multiantennas in the sensor nodes. Meanwhile, recent advances in hardware design allow MIMO systems to be integrated into a wireless sensor node [3]. All these development and advantages justify the merits of MIMO sensor systems.

In this paper, we consider the cross-layer optimization problem of physical and network layers to achieve performance enhancement on both transmission reliability and energy conservation. Joint channel coding, energy allocation and route planning are studied, based on which, a reliable packet forwarding scheme with optimized energy utilization is proposed. Our work mainly focuses on four aspects: 1) devise an efficient routing protocol; 2) adaptively allocate the transmission power; 3) maximize the STR, and 4) satisfy the total power constraint. Specifically, we formulate the joint energy and route optimization problem respectively as deterministic and stochastic optimization problems, and point out that a localized packet forwarding problem could be treated as a typical stochastic optimization problem. The optimization is then separated into two phases: route construction and energy reallocation. A low-complexity solution is then presented to reduce the computational complexity by decoupling the decision components of relaying nodes and energy budgets.

The rest of this paper is organized as follows. The system model is introduced in section II, where the network architecture, nodal requirements and wireless channel model are provided. The problem formulation is presented in section III. The globalized and localized routing problems are both taken into account. Section IV discusses the solution, which reduces the computational complexity by decomposing the decision components. Section V reports the results of the simulation results to demonstrate the effectiveness of our scheme. The conclusions are given in section VI.

\section{System Model}

\section{A. Network and Node Models}

Consider a self-organized wireless sensor network (WSN) in a static planar architecture. There is a data center in the network, responsible for data fusion and network maintenance. Let $\mathcal{V}$ denote the set of all sensor nodes and $\mathcal{L}$ denote the set of all links. A WSN is modelled as an undirected graph $G(\mathcal{V}, \mathcal{L})$. For $\forall v \in \mathcal{V}, S(v)$ is defined as the one-hop neighborhood of node $v$, which is the reachable (coverage) area of $v$ by using its maximum transmitting power. For $\forall v_{i}, v_{j} \in \mathcal{V}$, link $l=\left(v_{i}, v_{j}\right) \in \mathcal{L}$ if and only if $v_{j} \in S\left(v_{i}\right)$ [or equivalently, $v_{i} \in S\left(v_{j}\right)$ ]. A WSN routing problem is 
described as the problem to find a routing path consisting of a set of nodes $\left\{v_{0}, \cdots, v_{i}, v_{i+1}, \cdots, v_{N}\right\}$ with $\left(v_{i}, v_{i+1}\right) \in \mathcal{L}$, $i=0,1, \cdots, N$, which connects the source node $v_{0}$ and the destination node $v_{N}$ and satisfies performance requirements.

Each sensor node is assumed to know its position information. Recent availability of small-sized, low power GPS receivers and position estimation techniques justify the efficiency and feasibility of position-based routing protocols [4]. We also supposed that nodes are deployed uniformly at random in the plane region [5]. The randomness of the nodal position could be described as Poisson distribution. Nodes could exchange position information with their immediate neighboring nodes. The data center has the knowledge of the nodal density and the whole region size. We also assume that each sensor node is equipped with $N_{t}$ isotropic transmitting antennas and $N_{r}$ isotropic receiving antennas.

\section{B. Wireless Channel Model and Reliability Function}

We suppose that wireless channel suffers slow and flat Rayleigh fading without multipath fading or shadowing. Sensor nodes are sufficiently separated so that any mutual coupling effects among the antennas of different nodes are negligible.

The transmit symbol vector of size $K \times 1$ is denoted $\mathbf{x}=\left[x_{1}, \ldots, x_{K}\right]^{T}$, where $x_{i} \in \mathcal{A}$, where $\mathcal{A}$ is a signal constellation set. The vector $\mathbf{x}$ is transmitted by means of a given OSTBC matrix $\mathbf{C}(\mathbf{x})$ of size $B \times N_{t}$, where $B$ and $N_{t}$ are the space and time dimension of the OSTBC, respectively. If bits are used as inputs to the system, $K \log _{2} M$ bits are used to produce the vector $\mathbf{x}$. In [2], the following holds: $\mathbf{C}(\mathbf{x}) \mathbf{C}(\mathbf{x})^{H}=\beta K \mathbf{I}_{B}$, where $\beta=1$ if $\mathbf{C}(\mathbf{x})=\mathcal{G}_{2}^{T}$, $\mathbf{C}(\mathbf{x})=\mathcal{H}_{3}^{T}$ or $\mathbf{C}(\mathbf{x})=\mathcal{H}_{4}^{T}$ and $\beta=2$ if $\mathbf{C}(\mathbf{x})=\mathcal{G}_{3}^{T}$ or $\mathbf{C}(\mathbf{x})=\mathcal{G}_{4}^{T}$. The transmission rate is $N_{t} / B$, and the $\mathbf{C}(\mathbf{x})$ is sent over the MIMO channel $\mathbf{H}$ of site $N_{r} \times B$.

At the $i$-th hop, the $N_{t} \times N_{r}$ receive block signal $\mathbf{Y}$ can be written as

$$
\mathbf{Y}=l_{i}^{-\alpha} \mathbf{H C}(\mathbf{x})+\mathbf{N},
$$

where $l_{i}$ represents the communication distance of the $i$-th hop, $\alpha$ denotes the path loss component, and the additive block noise $\mathbf{N}$ is complex Gaussian circularly distributed with independent components having variance $N_{0}$ and zero mean.

By generalizing the approach given in [6], the OSTBC system can be shown to be equivalent to a SISO system with the following input output relationship

$$
y_{k}=\sqrt{\varphi} l_{i}^{-\alpha} x_{k}+n_{k},
$$

where $k \in\{1, \ldots, K\}, \varphi \triangleq\|\mathbf{H}\|^{2}$, and $n_{k} \sim \mathcal{C N}\left(0, N_{0} / \beta\right)$. It can be seen that the receive signal to noise ratio (SNR) $\gamma_{i}$ for a particular realization of the fading is given by $\gamma_{i} \triangleq \frac{p_{i}^{\text {tx }} \beta \varphi}{l_{i}^{2 \alpha} N_{0}}$, where $p_{i}^{\mathrm{tx}}$ is the transmitting power. Generally, $p_{i}^{\mathrm{tx}}=p_{i}-p_{i}^{\mathrm{c}}$ where $p_{i}$ is the total energy consumption of the $i$-th hop and $p_{i}^{\mathrm{c}}$ is the energy consumption inside the node. At the $i$-th hop, for a given $\gamma_{i}$, the corresponding symbol error rate (SER) is determined by [7]

$$
\operatorname{SER}\left(\gamma_{i}\right)=\frac{1}{\pi} \int_{0}^{\frac{(M-1) \pi}{M}} \exp \left(-\frac{g_{\mathrm{psk}} \gamma_{i}}{\sin ^{2} \theta}\right) d \theta
$$

where $g_{\mathrm{psk}} \triangleq \sin ^{2} \frac{\pi}{M}$. By averaging (3) over the channel, we obtain SER as a function of $l_{i}$ and $p_{i}$ [2] [7]

$$
\operatorname{SER}\left(l_{i}, p_{i}\right)=\frac{1}{\pi} \int_{0}^{\frac{(M-1) \pi}{M}}\left[1+\frac{g_{\mathrm{psk}} \beta}{N_{0} \sin ^{2} \theta}\left(p_{i}-p_{i}^{\mathrm{c}}\right) l_{i}^{-2 \alpha}\right]^{-N_{t} N_{r}} d \theta \text {. }
$$

The successful transmission rate (STR) for $x_{k}$ is given by

$$
\operatorname{STR}\left(l_{i}, p_{i}\right)=1-\operatorname{SER}\left(l_{i}, p_{i}\right) .
$$

Finally, we define reliability function $F_{R}$ as end-to-end STR

$$
F_{R}=\prod_{i=1}^{N} \operatorname{STR}\left(l_{i}, p_{i}\right)
$$

\section{Problem Formulation}

The joint energy and routing optimization problem is to build up an end-to-end routing path and to find an energy allocation scheme, which maximizes the STR while satisfying the energy consumption requirement. In this section, we formulate the joint energy and routing problem respectively from the point of view of globalized routing and localized routing.

\section{A. Globalized Routing Problem}

Consider a wireless sensor network $G(\mathcal{V}, \mathcal{L})$ and an endto-end routing path $\left\{v_{0}, \cdots, v_{i}, \cdots, v_{N}\right\}, v_{i} \in \mathcal{V}, i=$ $0,1, \cdots, N$, where $v_{0}$ and $v_{N}$ respectively stand for the source and the destination nodes. Let $l_{i}$ denote the $i$-th link $\left(v_{i-1}, v_{i}\right)$, and $p_{i}$ the corresponding energy consumption of the $i$-th hop. Let $L=\left\{l_{1}, \cdots, l_{N}\right\}$ and $P=\left\{p_{1}, \cdots, p_{N}\right\}$ respectively denote the link and the energy consumption sets of the forwarding scheme. The joint energy and route optimization problem can be formulated as

$$
\begin{aligned}
\max _{L \subset \mathcal{L}, P \subset \mathbb{R}} & F_{R}(L, P)=\prod_{i=1}^{N} \operatorname{STR}\left(l_{i}, p_{i}\right) \\
\text { s.t. } & \sum_{i=1}^{N} p_{i} \leq P_{c} \\
& p_{i} \geq 0,(i=1, \cdots, N),
\end{aligned}
$$

where $\operatorname{STR}\left(l_{i}, p_{i}\right)$ is the STR of the $i$-th hop given by (5), and $P_{c}$ is the energy constraint for end-to-end transmissions.

\section{B. Localized Routing Problem}

For distributed implementation, we reformulate (7) as a localized routing problem. We first introduce the relay-selecting rule to facilitate the localized routing. After that, we describe the problem in the form of stochastic optimization.

Relay-selection rule describes the instructions to select the relaying nodes. It could be carried out hop by hop in a distributed manner. Considering the $i$-th hop as shown in Fig. 1, the following definitions are introduced.

- Target position: In order to determine the relaying node $v_{i}$, node $v_{i-1}$ firstly assigns a target position $\hat{v}_{i}$, indicating the ideal position of $v_{i}$. Target position $\hat{v}_{i}$ will always appear on the line connecting $v_{i-1}$ and destination $v_{N}$. 


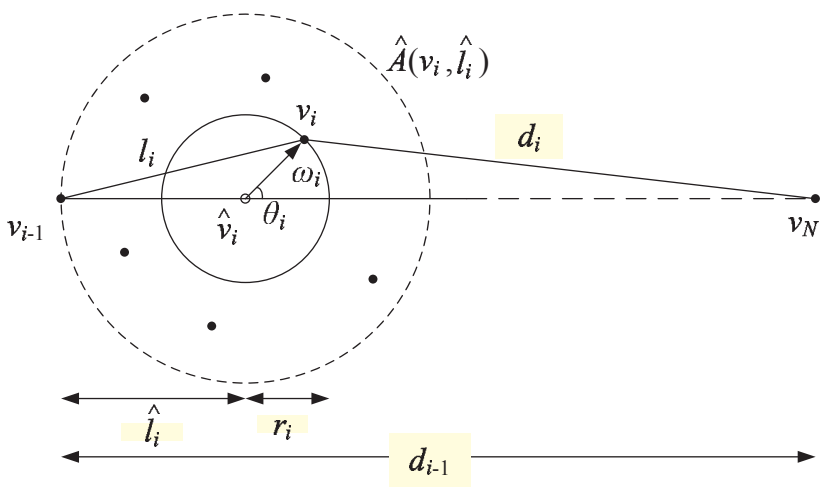

Fig. 1. One-hop relaying model.

- Target advancement: The distance from node $v_{i-1}$ to the ideal position $\hat{v}_{i}$ is called the target advancement of the $i$-th hop, denoted by $\hat{l}_{i}=\left(v_{i-1}, \hat{v}_{i}\right)$.

- Relay-selection area: Given an ideal position $\hat{v}_{i}$ and the target advancement $\hat{l}_{i}$, the disk area centering at $\hat{v}_{i}$ and with radius $\hat{l}_{i}$ is called the relay-selection area of the $i$-th hop, denoted by $A\left(\hat{v}_{i}, \hat{l}_{i}\right)$. To avoid backward forwarding, we restrict that node $v_{i}$ should locate within $A\left(\hat{v}_{i}, \hat{l}_{i}\right)$.

- Deviation vector: The vector from $\hat{v}_{i}$ to $v_{i}$ is called the deviation vector, denoted by $\omega_{i}$. Due to the uncertainty of node distribution, deviation vector $\omega_{i}$ is actually a random vector. We introduce polar coordinate and denote the deviation vector by two-tuple variables $\left(r_{i}, \theta_{i}\right)$.

There is a geometric relationship among the target advancement $\hat{l}_{i}$, the deviation vector $\omega_{i}$ [or equivalently $\left(r_{i}, \theta_{i}\right)$ ] and link $l_{i}$

$$
l_{i}=\sqrt{\hat{l}_{i}^{2}+r_{i}^{2}+2 \hat{l}_{i} r_{i} \cos \theta_{i}} .
$$

For the sake of presentation, we let $g\left(\hat{l}_{i}, \omega_{i}\right)=$ $\sqrt{\hat{l}_{i}^{2}+r_{i}^{2}+2 \hat{l}_{i} r_{i} \cos \theta_{i}}$ hereafter.

The relay-selection rule works as follows: at the $i$-th hop, node $v_{i-1}$ should first specify the target advancement $\hat{l}_{i}$ and the target position $\hat{v}_{i}$. After that, node $v_{i-1}$ selects a neighboring node within relay-selecting area $A\left(\hat{v}_{i}, \hat{l}_{i}\right)$ as the relaying node $v_{i}$, which is the closest to position $\hat{v}_{i}$.

The relay-selecting rule facilitates the localized routing by regulating the basic operations for a node to determine its next relaying node. According to relay-selection rule, the exact positions of the relaying nodes are determined by both the routing decisions (i.e., the target advancements $\hat{l}_{i}$ 's), and the uncertain factors (i.e., the random deviation vectors $\omega_{i}$ 's). The end-to-end STR, however, varies with these uncertain factors. It is more meaningful to redefine the reliability function as the average end-to-end STR. Let $\hat{L}=\left\{\hat{l}_{1}, \cdots, \hat{l}_{N}\right\}$ denote the set of target advancements and $\Omega=\left\{\omega_{1}, \cdots, \omega_{N}\right\}$ the set of deviation vectors. The localized routing problem can then be redescribed as the following stochastic optimization.

$$
\begin{aligned}
\max _{\hat{L} \subset \mathbb{R}, P \subset \mathbb{R}} & F_{R}(\hat{L}, P)=\mathbf{E}_{\Omega}\left[\prod_{i=1}^{N} \operatorname{STR}\left(g\left(\hat{l}_{i}, w_{i}\right), p_{i}\right)\right] \\
\text { s.t. } & \sum_{i=1}^{N} p_{i} \leq P_{c} \\
& p_{i} \geq 0,(i=1, \cdots, N) .
\end{aligned}
$$

Since the distribution of nodes' positions can be described by Poisson model [5], the Probability Density Function (PDF) of deviation vectors is computable, and therefore the reliability function is predictable.

\section{LOW-COMPLEXITY SOLUTION}

As shown in problem (9), the objective is to find the optimized target advancements $\hat{l}_{i}$ 's and the energy budgets $p_{i}$ 's. In this paper, we develop a low-complexity suboptimal solution, which reduces the complexity by decoupling the variables $\hat{l}_{i}$ 's and $p_{i}$ 's. We decompose the joint optimization process into two separate phases as explained below.

- Route construction: Assuming that all hops are allocated equal energy, relaying nodes can be selected hop by hop using relay-selection rule.

- Energy reallocation: Given an end-to-end route, the source node performs the energy reallocation to maximize the end-to-end STR.

At the beginning of the first phase, the source node computes the optimal total hop count $N^{*}$. After that, relaying nodes are selected hop by hop. For the $i$-th hop, the distance of $\hat{l}_{i}$ is set to $\frac{d_{i-1}}{N^{*}-i+1}$ (see Fig. 1). Node $v_{i}$ is determined by the relay-selecting rule. In the second phase, source will perform energy reallocation by linear programming. The main operations of the solution are discussed in the following.

\section{A. Optimal Hop Count Determination}

In the first phase, the source node computes the optimal hop count $N^{*}$ in two steps: i) computing average STR per hop, and ii) approximating average STR of the entire route.

1) Average STR per hop: Supposing that the hop count is $N$, we investigate the $i$-th hop as shown in Fig. 1. Let $f_{\omega}\left(r_{i}, \theta_{i}\right)$ represent the PDF of deviation vector $\omega$ of the $i$-th hop, and $\overline{S T R}\left(\hat{l}_{i}, p\right)$ the average STR of the $i$-th hop. Following the relay-selecting rule, we have

$$
\begin{aligned}
\overline{S T R}\left(\hat{l}_{i}, p\right) & =\mathbf{E}_{\omega_{i}}\left[\operatorname{STR}\left(l_{i}, p\right)\right] \\
& =\int_{0}^{\hat{l}_{i}} \int_{0}^{2 \pi} \operatorname{STR}\left(l_{i}, p\right) f_{\omega}\left(r_{i}, \theta_{i}\right) r_{i} d \theta_{i} d r_{i},
\end{aligned}
$$

where $\hat{l}_{i}=\frac{d_{i-1}}{N-i+1}, p=\frac{P_{c}}{N}$ and $l_{i}$ is given by (8). Note that, when sensors are deployed uniformly at random in the field, the explicit form of $f_{\omega}(r, \theta)$ could be obtained by Poisson Process model. The detailed derivation is shown in the appendix. 
2) Average STR of entire path: Let $\Omega_{k}=\left\{\omega_{1}, \omega_{2}, \cdots, \omega_{k}\right\}$, $k=1, \cdots, N$, and specially $\Omega=\Omega_{N}$. The average STR of the entire path is given by

$$
\overline{S T R}=\mathbf{E}_{\Omega}\left[\prod_{i=1}^{N} \overline{S T R}\left(\hat{l}_{i}, p\right)\right] .
$$

Since actual STR is usually very close to one, the logarithm function is almost linear. We are allowed to exchange the operation order of expectation and logarithm. Then, we have

$$
\begin{aligned}
\log (\overline{S T R}) & \simeq \mathbf{E}_{\Omega}\left\{\log \left[\prod_{i=1}^{N} \overline{S T R}\left(\hat{l}_{i}, p\right)\right]\right\} \\
& =\sum_{i=1}^{N} \mathbf{E}_{\Omega_{i}}\left\{\log \left[\overline{S T R}\left(\hat{l}_{i}, p\right)\right]\right\} \\
& \simeq \sum_{i=1}^{N} \log \left\{\mathbf{E}_{\Omega_{i}}\left[\overline{S T R}\left(\hat{l}_{i}, p\right)\right]\right\} .
\end{aligned}
$$

By computing exponential function on both sides of (12), we have

$$
\overline{S T R} \simeq \prod_{i=1}^{N} \mathbf{E}_{\Omega_{i}}\left[\overline{S T R}\left(\hat{l}_{i}, p\right)\right],
$$

where the average STR of the entire path is the product of the average STR of all hops.

Note that $\hat{l}_{i}=\frac{d_{i-1}}{N-i+1}$ and $\mathbf{E}_{\Omega_{i}}\left[\overline{S T R}\left(\hat{l}_{i}, p\right)\right]=$ $\mathbf{E}_{\Omega_{i}}\left[\overline{S T R}\left(\frac{d_{i-1}}{N-i+1}, p\right)\right]$. The accurate computation of $\mathbf{E}_{\Omega_{i}}\left[\overline{S T R}\left(\frac{d_{i}}{N-i}, p\right)\right]$ is considerably complex when $i$ is large. To show this, let us consider the iterative relationship of $d_{i}$ and $d_{i-1}$ as shown in Fig. 1.

$$
d_{i}=\sqrt{r_{i}^{2}+\left(d_{i-1}-\hat{l}_{i}\right)^{2}-2 r_{i}\left(d_{i-1}-\hat{l}_{i}\right) \cos \theta_{i}} .
$$

For convenience, we let $h\left(d_{i-1}, \omega_{i}\right)=$ $\sqrt{r_{i}^{2}+\left(d_{i-1}-\hat{l}_{i}\right)^{2}-2 r_{i}\left(d_{i-1}-\hat{l}_{i}\right) \cos \theta_{i}}$, where the deviation vector $\omega_{i}=\left(r_{i}, \theta_{i}\right)$. It is shown in (14) that the value of $d_{i}$ depends on the value of $d_{i-1}$ as well as the deviation vector $\hat{\omega}_{i}$. The derivation of the PDF of $d_{i}$ is explicitly related to the PDF of $d_{i-1}$, and then implicitly to the PDFs of $d_{i-2}, d_{i-3}, \cdots, d_{1}$. Therefore as $i$ increases, the computation of PDF of $d_{i}$ becomes nontrivial, and the accurate computation of $\mathbf{E}_{\Omega_{i}}\left[\overline{S T R}\left(\frac{d_{i}}{N-i}, p\right)\right]$ becomes too complicated to be carried out in practical sensor nodes. Alternatively, we adopt the following approximation

$$
\mathbf{E}_{\Omega_{i}}\left[\overline{S T R}\left(\frac{d_{i}}{N-i}, p\right)\right] \simeq \overline{S T R}\left(\frac{\mathbf{E}_{\Omega_{i}}\left[d_{i}\right]}{N-i}, p\right),
$$

where the expectation of $\overline{S T R}\left(\frac{d_{i}}{N-i}, p\right)$ over $\Omega_{i}$ is approximated by the value of function $\overline{S T R}(\cdot, p)$ at the single point $\frac{\mathbf{E}_{\Omega_{i}}\left[d_{i}\right]}{N-i}$. We compute expectation on both sides of (14) and obtain

$$
\begin{aligned}
\mathbf{E}_{\Omega_{i}}\left[d_{i}\right] & =\mathbf{E}_{\Omega_{i}}\left[h\left(d_{i-1}, \omega_{i}\right)\right] \\
& \simeq \mathbf{E}_{\omega_{i}}\left\{h\left(\mathbf{E}_{\Omega_{i-1}}\left[d_{i-1}\right], \omega_{i}\right)\right\} .
\end{aligned}
$$

Similar as (15), we replace the expectation of $h\left(d_{i-1}, \omega_{i}\right)$ over $\Omega_{i-1}$ by the value of function $h\left(\cdot, \omega_{i}\right)$ at the point $\mathbf{E}_{\Omega_{i-1}}\left[d_{i-1}\right]$.
Actually, (16) provides an approximative iteration of $\mathbf{E}_{\Omega_{i}}\left[d_{i}\right]$, which could be extended to

$$
\mathbf{E}_{\Omega_{i}}\left[d_{i}\right]=\int_{0}^{l_{i}^{\prime}} \int_{0}^{2 \pi} h\left(\mathbf{E}_{\Omega_{i-1}}\left[d_{i-1}\right], \omega_{i}\right) f_{\omega}\left(r_{i}, \theta_{i}\right) r_{i} d \theta_{i} d r_{i}
$$

where $l_{i}^{\prime}=\frac{\mathbf{E}_{\Omega_{i-1}}\left[d_{i-1}\right]}{N-i+1}$ is the average target advancement of the $i$-th hop

\section{B. Routing Path Determination}

After computing the optimal hop count $N^{*}$, the source node launches the process of routing path determination. Starting from the source node and hop by hop sequentially, a current node selects the relaying node and forwards the packet by the relay-selection rule. Each hop consumes energy $p=\frac{P_{c}}{N^{*}}$. The operations go on until the packet reaches node $v_{N}$.

When running relay-selection rule, it is possible that no sensor locates in the relay-selection area. To deal with this situation, an additional rule should be followed: when node $v_{i-1}$ fails to find a relaying node within the relay-selection area $A\left(\hat{v}_{i}, \hat{l}_{i}\right)$, it should reduce the total hop count by $N=$ $N-1$, and recompute the target position $\hat{v}_{i}$ and the target advancement $\hat{l}_{i}$. Node $v_{i-1}$ should try to reselect the relaying node $v_{i}$ within the new $A\left(\hat{v}_{i}, \hat{l}_{i}\right)$. Nevertheless, this situation happens very infrequently in practice. For instance, supposing the nodal density is $0.1 / \mathrm{m}^{2}$, this happens only with probability $3.8 \times 10^{-4}$ for $\hat{l}_{i}=5 \mathrm{~m}$, and $1.8 \times 10^{-9}$ for $\hat{l}_{i}=8 \mathrm{~m}$.

\section{Energy Reallocation}

After the route is established, the source node will reallocate the energy consumption as the optimization problem below.

$$
\begin{aligned}
\max & \sum_{i=1}^{N} S T R_{i}^{\prime}\left(l_{i}, p_{i}\right), \\
\text { s.t. } & \sum_{i=1}^{N} p_{i} \leq P_{c} \\
& p_{i} \geq 0,(i=1, \cdots, N) .
\end{aligned}
$$

where $S T R^{\prime}=\log (S T R)$ and $l_{i}$ 's are given. In order to find $p_{i}$ 's such that the $S T R^{\prime}$ is maximized. We consider

$$
L\left(p_{1}, \cdots, p_{N}\right)=\sum_{i=1}^{N} S T R_{i}^{\prime}-\lambda\left(\sum_{i=1}^{N} p_{i}-P_{c}\right),
$$

where $\lambda$ is a positive Lagrange multiplier. The necessary condition for optimality can be obtained by finding the derivatives of (19) with respect to $p_{i}$ 's. For nodes $v_{i}$ 's with nonzero consumption energy, we have

$$
\frac{\partial L\left(p_{1}, \cdots, p_{N}\right)}{\partial p_{i}}=\frac{\partial S T R_{i}^{\prime}}{\partial p_{i}}-\lambda,
$$

where

$$
\begin{aligned}
& \frac{\partial S T R_{i}^{\prime}}{\partial p_{i}}=\frac{1}{1-S E R_{i}} \frac{\partial S E R_{i}}{\partial p_{i}} \\
& =\frac{g_{\mathrm{psk}} \beta d_{i}^{-2 \alpha}}{\left(S E R_{i}-1\right) N_{0} \pi} \int_{0}^{\frac{(M-1) \pi}{M}} \sin ^{-2} \theta\left(1+\frac{g_{\mathrm{psk}} \beta}{N_{0} \sin ^{2} \theta} p_{i} d_{i}^{-2 \alpha}\right)-2 d \theta .
\end{aligned}
$$


Using (20) and (21), the optimal energy budget at each hop can be calculated by the gradient descent optimization method [8]

$$
\begin{aligned}
p_{i}(k+1) & =p_{i}(k)-\mu \frac{\partial L\left(p_{1}, \ldots, p_{N}\right)}{\partial p_{i}} \\
& =p_{i}(k)-\mu\left(\frac{\partial S T R_{i}^{\prime}}{\partial p_{i}}-\lambda(k)\right),(i=1, \cdots, N),
\end{aligned}
$$

where $p_{i}(k)$ and $\lambda(k)$ represent the transmission power and Lagrangian multiplier at the $k$-th iteration, and $\mu$ stands for the positive step size. Using the power constraint, $\lambda(k)$ can be obtained by the following equation

$$
\sum_{i=1}^{N} p_{i}(k+1)-P_{c}=0 .
$$

The energy reallocation scheme can be easily solved by initializing some positive values for $p_{i}(i=1, \cdots, N)$ and using (22) in an iterative manner.

\section{Numerical Results}

This section evaluates the proposed packet forwarding scheme by simulation results. In the simulation, we consider a square field with area $100 \times 100 \mathrm{~m}^{2}$. Sensor nodes are uniformly deployed at random in the region with nodal density $0.1 / \mathrm{m}^{2}$. Wireless bandwidth is supposed to be $1 \mathrm{MHz}$. Packet size is chosen to be $1 \mathrm{kbs}$. Parameters in the reliability function are set as follows: number of transmit antennas $N_{t}=2$, number of receive antennas $N_{r}=2$, constellation size $M=2$, attenuation coefficient $\alpha=3$, OSTBC matrix $\mathbf{C}(\mathbf{x})=\mathcal{G}_{2}^{T}$, $\beta=1$, normalized energy consumption inside the nodes $p^{c}=1200$, normalized noise power $N_{0}=1$. Besides the proposed scheme, we also simulate two traditional energyefficient routing algorithms for comparison: SP-power [4] and PARO [9]. In the figures, the proposed scheme, SP-power and PARO are labelled as $L P, S P R$ and $P A R O$, respectively.

We first validate the theoretical computation of optimal hop count $N^{*}$. In particular, given the source and destination nodes and the energy constraint, we investigate the relationship between hop count $N$ and the average end-to-end STR. Three cases are considered where the energy constraints $P_{c}$ are fixed by $5 \times 10^{4}$ and the distances of source and destination nodes

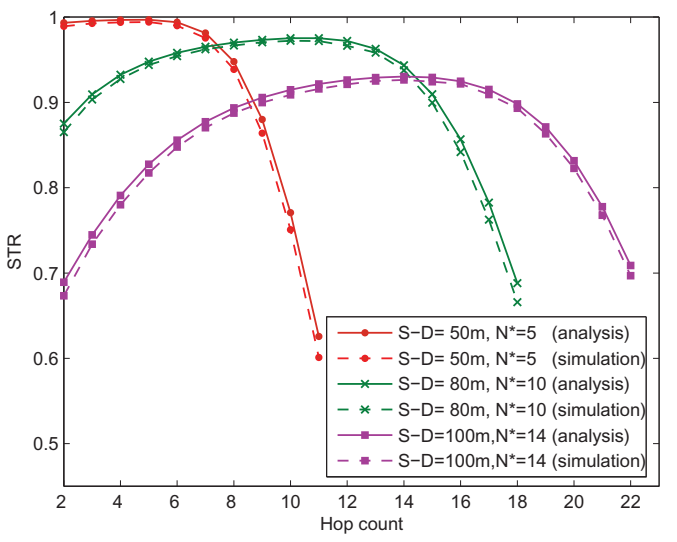

Fig. 2. Average STR vs. hop count.

are set to be $100 \mathrm{~m}, 80 \mathrm{~m}$ and $50 \mathrm{~m}$, respectively. As shown in Fig. 2, the curves of theoretical computation and simulation results are very close in all cases. The theoretical optimal hop count (i.e., 14 for $\mathrm{S}-\mathrm{D}=100 \mathrm{~m}, 10$ for $\mathrm{S}-\mathrm{D}=80 \mathrm{~m}$ and 5 for $\mathrm{S}-\mathrm{D}=50 \mathrm{~m}$ ) exactly equals to the one that will generate maximum STR in the simulation. This verifies the correctness of the optimal hop count computation.

Table I reports the details of the linear programming for energy reallocation. We conduct five individual simulations, where the energy constraint $E_{c}$ is fixed by $5 \times 10^{4}$ and the S-D distances are set to $100 \mathrm{~m}, 90 \mathrm{~m}, 80 \mathrm{~m}, 70 \mathrm{~m}$ and $60 \mathrm{~m}$ respectively. The actual link distances, energy budgets and total energy consumption are recorded. As shown in table I, although $E_{c}$ is fixed, the proposed scheme could adaptively regulate the energy budgets of all hops according to the actual link distances. The total energy consumptions strictly follow the constraints in all simulations.

Fig. 3 and Fig. 4 provide quantitative results to compare the reliability performance of the three schemes. We investigate the influences of S-D distance and energy constraint to the endto-end STR. In Fig. 3, the energy constraint is fixed as $5 \times 10^{4}$ while in Fig. 4, the S-D distance is fixed as $50 \mathrm{~m}$. We change the S-D distance in Fig. 3 and energy constraint in Fig. 4, and keep track of the end-to-end STR. As shown in Fig. 3 and Fig. 4, the proposed scheme clearly outperforms the traditional

\begin{tabular}{|c|c|c|c|c|c|c|c|c|c|c|c|c|c|c|c|c|c|c|c|c|c|}
\hline S-D & Para. Link & $l_{1}$ & $l_{2}$ & $l_{3}$ & $l_{4}$ & $l_{5}$ & $l_{6}$ & $l_{7}$ & $l_{8}$ & $l_{9}$ & $l_{10}$ & $l_{11}$ & $l_{12}$ & $l_{13}$ & $l_{14}$ & $l_{15}$ & $l_{16}$ & $l_{17}$ & $l_{18}$ & $l_{19}$ & Total \\
\hline \multirow{2}{*}{$100 \mathrm{~m}$} & Distance $(m)$ & 3.3 & 7.6 & 4.3 & 6.5 & 5.0 & 7.8 & 5.3 & 6.1 & 3.7 & 5.5 & 4.0 & 5.2 & 6.0 & 5.1 & 4.2 & 4.3 & 6.3 & 5.5 & 6.7 & \\
\hline & Energy $\left(\times 10^{3}\right)$ & 1.8 & 2.9 & 2.4 & 2.9 & 2.7 & 2.9 & 2.8 & 2.9 & 2.1 & 2.8 & 2.2 & 2.7 & 2.9 & 2.7 & 2.3 & 2.4 & 2.9 & 2.8 & 2.9 & 49.9 \\
\hline \multirow[b]{2}{*}{$90 m$} & Distance $(m)$ & 2.0 & 7.5 & 6.0 & 4.1 & 6.3 & 5.2 & 5.0 & 6.3 & 5.2 & 6.3 & 7.6 & 7.5 & 4.2 & 6.3 & 2.5 & 6.5 & 6.1 & & & \\
\hline & Energy $\left(\times 10^{3}\right)$ & 1.3 & 3.3 & 3.3 & 2.5 & 3.3 & 3.1 & 3.0 & 3.3 & 3.1 & 3.3 & 3.3 & 3.3 & 2.6 & 3.3 & 1.4 & 3.3 & 3.3 & & & 50.0 \\
\hline \multirow{2}{*}{$80 m$} & Distance $(m)$ & 6.3 & 6.4 & 4.3 & 4.6 & 5.6 & 5.9 & 6.1 & 5.0 & 5.8 & 5.9 & 5.1 & 7.1 & 2.7 & 6.1 & 7.0 & & & & & \\
\hline & Energy $\left(\times 10^{3}\right)$ & 3.6 & 3.6 & 2.9 & 3.1 & 3.5 & 3.6 & 3.6 & 3.3 & 3.6 & 3.6 & 3.4 & 3.6 & 1.5 & 3.6 & 3.6 & & & & & \\
\hline \multirow{2}{*}{$70 m$} & Distance $(m)$ & 4.5 & 4.9 & 3.9 & 5.9 & 4.6 & 7.2 & 4.7 & 6.2 & 4.9 & 6.3 & 4.6 & 8.3 & 5.7 & & & & & & & \\
\hline & Energy $\left(\times 10^{3}\right)$ & 3.5 & 3.8 & 3.0 & 4.2 & 3.6 & 4.2 & 3.7 & 4.2 & 3.8 & 4.2 & 3.6 & 4.1 & 4.1 & & & & & & & 49 \\
\hline \multirow{2}{*}{$60 \mathrm{~m}$} & Distance $(m)$ & 5.4 & 4.3 & 8.9 & 4.6 & 6.2 & 7.8 & 4.8 & 5.5 & 4.9 & 5.1 & 6.0 & & & & & & & & & \\
\hline & Energy $\left(\times 10^{3}\right)$ & 4.7 & 3.8 & 4.6 & 4.2 & 5.0 & 4.8 & 4.3 & 4.8 & 4.4 & 4.5 & 4.9 & & & & & & & & & 50.0 \\
\hline
\end{tabular}

TABLE I

ENERGY BUDGETS IN LINEAR PROGRAMMING $\left(P_{c}=5 \times 10^{4}\right)$ 


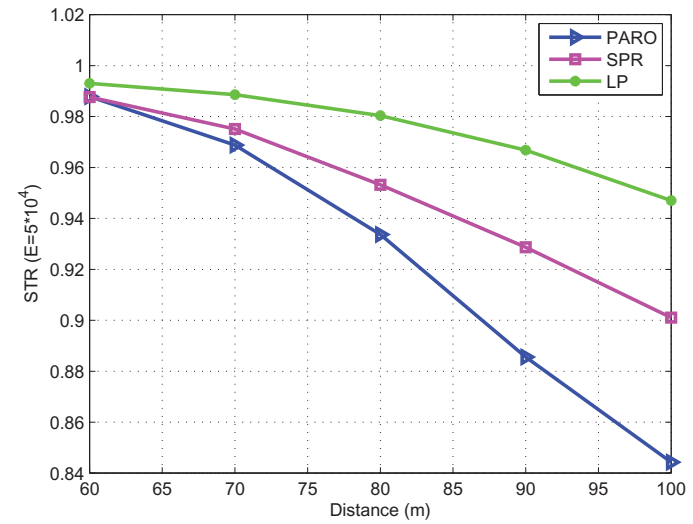

Fig. 3. STR performance in term of S-D distance.

algorithms, especially when the S-D distance is large or the energy constraint is stringent. It is noticeable that, the proposed scheme has an enhancement of end-to-end STR at least $10 \%$ in Fig. 3 when the S-D distance is $100 \mathrm{~m}$, and about $2 \%$ in Fig. 4 when the energy constraint is $3 \times 10^{4}$. These results are expectable because the proposed scheme simultaneously takes into account of the issues of transmission reliability and energy conservation. It aims at maximizing the reliability by fully exploiting the limited energy resource.

\section{CONCLUSION}

In this paper, we propose a cross-layer optimized forwarding scheme for reliable transmissions in WSNs. Each sensor has multiple antennas using OSTBC codes. Channel coding, power allocation and route planning are jointly optimized to maximize the end-to-end STR while satisfying the energy constraint. The problem is formulated as a localized optimization problem. By decoupling the components of forwarding decision, we propose a low-complexity suboptimal solution. Simulation is carried out to evaluated the proposed forwarding scheme. The results validate the effectiveness of the proposed scheme, and show that it significantly outperforms two traditional energy-efficient routing algorithms.

\section{APPENDIX A}

\section{Derivation of Closed-Form Expression of $f_{\omega}(r, \theta)$}

We present in this appendix the derivation on the explicit expression of transition PDF of the deviation vector $f_{\omega}(\cdot, \cdot)$. As mentioned before, sensors are supposed to be deployed uniformly at random in the region of interest. Poisson Process model is appropriate to describe the node distribution [5]. As shown in Fig. 1, let $\omega=(r, \theta)$ and $\hat{l}$ respectively denote the deviation vector and the target advancement. By relayselecting rule, we have

$$
\operatorname{Pr}[r \leq z]= \begin{cases}1-e^{-\rho \pi z^{2}} & 0 \leq z \leq \hat{l} \\ 1 & z>\hat{l}\end{cases}
$$

where $\rho$ denotes the node density in the region. Note that we set $\operatorname{Pr}[r \leq z]=1$, when $z>\hat{l}$. The reason can be explained



Fig. 4. STR performance in term of energy constraint.

as follows: If there is no sensor within the relay-selecting area, the target advancement $\hat{l}$ is said to be improper, and a new target advancement has to be made (see section IV.B for details). The PDF of variable $r$ is given by

$$
f_{r}(z)=f_{r}^{\prime}(z)+\operatorname{Pr}[r=z] \delta(z-\hat{l}),
$$

where $f_{r}^{\prime}(z)$ is the derivative of $\operatorname{Pr}[r \leq z]$ when $z \in[0, \hat{l}]$, and $\operatorname{Pr}[r=z]=e^{-\rho \pi z^{2}}$. The PDF that $\omega$ occurs on position $(z, \theta)$ with target advancement $\hat{l}$ can be written as

$$
\begin{aligned}
f_{\omega}(z, \theta) & =\frac{1}{2 \pi z} f_{r}(z) \\
& =\rho e^{-\rho \pi z^{2}}+\frac{e^{-\rho \pi \hat{l}^{2}}}{2 \pi z} \delta(z-\hat{l}) \quad z \in[0, \hat{l}] .
\end{aligned}
$$

\section{ACKNOWLEDGEMENT}

The work in this paper is supported by programs of NSFC under Grant Nos. 60903170, U0835003, U0635001.

\section{REFERENCES}

[1] I. F. Akyildiz, W. Su, Y. Sankarasubramaniam, and E. Cayirci, "A Survey on Sensor Networks," IEEE Communications Magazine, vol. 40, no. 8 , pp. 102-114, August 2002.

[2] V. Tarokh, N. Seshadri, and A. R. Calderbank, "Space-Time Codes for High Data Rate Wireless Communication: Performance Criterion and Code Construction," IEEE Trans. Inform. Theory, vol. 44, no. 2, pp. 744 765, USA, Mar. 1998.

[3] S. Cui and A. Goldsmith, "Energy-Efficiency of MIMO and Cooperative MIMO Techniques in Sensor Networks," IEEE J. on Selected Areas in Commun., vol. 22, no. 6, pp. 1089-1098, Aug. 2004.

[4] I. Stojmenovic and X. Lin, "Power-Aware Localized Routing in Wireless Networks," IEEE Trans. Parallel Dist. Sys., vol.12, no.11, pp. 22-33, 2001.

[5] M. Zorzi and R. R. Rao, "Geographic Random Forwarding (GeRaF) for Ad Hoc and Sensor Networks: Multihop Performance," IEEE Trans. on Mobile Computing, vol. 2, no. 4, Oct.-Dec. 2003.

[6] H. Shin and J. H. Lee, "Exact Symbol Error Probability of Orthogonal Space-Time Block Codes," in Proc. IEEE GLOBECOM, vol. 2, pp. 11971201, Nov. 2002, USA.

[7] M. K. Simon and M.-S. Alouini, Digital Communications over Fading Channels: A Unified Approach to Performance Analysis. Wiley Series in Telecommunications and Signal Processing, USA, 2001.

[8] D. G. Luenberger, Linear and Nonlinear Programming, 2nd ed. AddisionWesley Publishing Company, Reading, Massachusetts, USA, 1984.

[9] J. Gomez, A.T. Campbell et al., "PARO: Conserving Transmission Power in Wireless Ad Hoc Networks," Proc. ICNP'01, pp. 24-34, 2001. 